\title{
Modelling resource requirements and physician staffing to provide virtual urgent medical care for residents of long-term care homes: a cross-sectional study
}

\author{
Fahad Razak MD MSc, Saeha Shin MPH, Frances Pogacar BCS, Hae Young Jung MSc, Laura Pus MBA, \\ Andrea Moser MD MSc, Lauren Lapointe-Shaw MD PhD, Terence Tang MD, Janice L. Kwan MD MPH, \\ Adina Weinerman MD MHSc, Shail Rawal MD MPH, Vladyslav Kushnir PhD, Denise Mak PhD, \\ Danielle Martin MD MPP, Kaveh G. Shojania MD, Sacha Bhatia MD MBA, Payal Agarwal MD, \\ Geetha Mukerji MD MSc, Michael Fralick MD PhD, Moira K. Kapral MD MSc, Matthew Morgan MD MSc, \\ Brian Wong MD, Timothy C.Y. Chan PhD, Amol A. Verma MD MPhil
}

\section{Abstract}

Background: The coronavirus disease 2019 (COVID-19) outbreak increases the importance of strategies to enhance urgent medical care delivery in long-term care (LTC) facilities that could potentially reduce transfers to emergency departments. The study objective was to model resource requirements to deliver virtual urgent medical care in LTC facilities.

Methods: We used data from all general medicine inpatient admissions at 7 hospitals in the Greater Toronto Area, Ontario, Canada, over a 7.5-year period (Apr. 1, 2010, to Oct. 31, 2017) to estimate historical patterns of hospital resource use by LTC residents. We estimated an upper bound of potentially avoidable transfers by combining data on short admissions ( $\leq 72 \mathrm{~h}$ ) with historical data on the proportion of transfers from LTC facilities for which patients were discharged from the emergency department without admission. Regression models were used to extrapolate future resource requirements, and queuing models were used to estimate physician staffing requirements to perform virtual assessments.

Results: There were 235375 admissions to general medicine wards, and residents of LTC facilities (age 16 yr or older) accounted for $9.3 \%$ ( $n=21948)$ of these admissions. Among the admissions of residents of LTC facilities, short admissions constituted $24.1 \%$ ( $n=5297)$, and for $99.8 \%(n=5284)$ of these admissions, the patient received laboratory testing, for $86.9 \%(n=4604)$ the patient received plain radiography, for $41.5 \%(n=2197)$ the patient received computed tomography and for $81.2 \%(n=4300)$ the patient received intravenous medications. If all patients who have short admissions and are transferred from the emergency department were diverted to outpatient care, the average weekly demand for outpatient imaging per hospital would be 2.6 ultrasounds, 11.9 computed tomographic scans and 23.9 radiographs per week. The average daily volume of urgent medical virtual assessments would range from 2.0 to 5.8 per hospital. A single centralized virtual assessment centre staffed by 2 or 3 physicians would provide services similar in efficiency (measured by waiting time for physician assessment) to 7 separate centres staffed by 1 physician each.

Interpretation: The provision of acute medical care to LTC residents at their facility would probably require rapid access to outpatient diagnostic imaging, within-facility access to laboratory services and intravenous medication and virtual consultations with physicians. The results of this study can inform efforts to deliver urgent medical care in LTC facilities in light of a potential surge in COVID-19 cases.

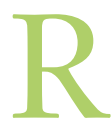
educing the transfer of residents of long-term care (LTC) facilities to acute care hospitals has been a focus of quality improvement efforts for many years. ${ }^{1}$ Coronavirus disease 2019 (COVID-19) has only sharpened this focus. COVID-19 affects older adults, especially those with chronic medical conditions, ${ }^{2}$ more severely than younger people, making LTC facilities an especially vulnerable setting for widespread transmission. Early examples of COVID-19 outbreaks in LTC facilities resulted from within-facility spread among residents and health care staff, with high fatality rates among residents. ${ }^{3}$ Nosocomial spread of COVID-19 from hospitals to LTC facilities could have dire consequences. ${ }^{3}$ A rapidly deployable strategy to reduce safely the frequency of transfers from LTC facilities to

Competing interests: None declared.

This article has been peer reviewed.

Correspondence to: Fahad Razak, fahad.razak@mail.utoronto.ca CMAJ Open 2020. DOI:10.9778/cmajo.20200098 
hospital emergency departments may reduce the risk of nosocomial exposure and improve capacity in emergency departments and hospital wards., 4,5 This would benefit patients and the health system more broadly.

The objective of this study was to support rapid deployment of creative interventions to deliver urgent medical care to LTC residents outside hospital settings, in the context of a widespread COVID-19 outbreak, by modelling resource requirements to delivery virtual urgent medical care in LTC facilities.

\section{Methods}

\section{Study design and population}

We used a cohort of patients admitted to the general internal medicine services of 7 large hospitals in Ontario to describe admissions to hospital of patients from LTC facilities and the subset of these admissions that were less than 72 hours; and the use of resources such as laboratory testing, imaging and intravenous (IV) medication among these patients. We then modelled the requirement for virtual consultations with specialist physicians and rapid access to outpatient diagnostic services if a program were implemented to provide urgent medical care in an LTC setting.

We used retrospective multicentre hospital data from the General Medicine Inpatient Initiative (GEMINI). Briefly, the GEMINI cohort comprises all patients admitted to or discharged from the general internal medicine service at 7 participating hospitals in Ontario (Sinai Health System [Mount Sinai Hospital] St. Michael's Hospital, University Health Network [Toronto General Hospital, Toronto Western Hospital], Sunnybrook Health Sciences Centre and Trillium Health Partners [Mississauga Hospital, Credit Valley Hospital]) in the Greater Toronto Area, home to more than 6 million people and the most populous metropolitan region in Canada. GEMINI hospitals include 5 academic institutions in Toronto ( 4 in the downtown core and 1 in a primarily residential region) and 2 community teaching hospitals in Mississauga. All participating hospitals are publicly funded, operate independently and provide tertiary and/or quaternary care.

In this study, we included all patients aged 16 years or older whose emergency department visit resulted in an admission between Apr. 1, 2010, and Oct. 31, 2017. We did not include patients who had an elective or planned hospital admission or patients who had an interhospital transfer. All patients were followed in hospital until death or discharge. We have previously described GEMINI in detail. ${ }^{6}$

\section{GEMINI data sources, methods and variables}

We collected data on patients' clinical and demographic characteristics from each hospital's electronic information systems and health administrative databases as reported to the Discharge Abstract Database and National Ambulatory Care Reporting System of the Canadian Institute for Health Information). ${ }^{7,8}$ Each inpatient and emergency department individual-level record included age, sex, place of residence at time of admission and discharge, most responsible discharge diagnosis, diagnostic and therapeutic procedures, laboratory (biochemistry, hematology and microbiology) test results, radiology test results, blood transfusions, medications and intensive care unit admissions. All data were linked using unique identifiers and a subset of the data demonstrated $98 \%-100 \%$ accuracy compared with chart review. ${ }^{9}$

We identified all patients who were admitted from LTC facilities using the institution number developed by the Ministry of Health and Long-Term Care Master Numbering System in our administrative data. ${ }^{10}$ These facilities included nursing homes and facilities offering complex continuing care but not rehabilitation hospitals.

We included the following patient and situational characteristics: age; sex; Charlson Comorbidity Index score calculated using the enhanced Canadian version of the International Statistical Classification of Diseases and Related Health Problems, 10th Revision (ICD-10-CA) and classified into groups with scores of $0-2$ and greater than $2 ;^{11}$ the Laboratory-based Acute Physiology Score (LAPS), which is a validated predictor of inpatient mortality risk; ${ }^{12}$ admission to the general internal medicine service at participating GEMINI hospitals in the 30 previous days; admission day and time; triage day and time; and calendar year of admission.

For each LTC-related admission, patient outcomes and resource utilization during hospital admission were characterized by examining the use of imaging procedures (radiography, computed tomography [CT], ultrasonography, magnetic resonance imaging [MRI] and interventional radiology procedures), erythrocyte transfusion and endoscopy..$^{13}$ We identified patients who were admitted to special care units (intensive care or "step-up" units), who died in hospital and who were readmitted to the general internal medicine service at participating GEMINI hospitals within 30 days of discharge. Furthermore, we reported the most responsible discharge diagnosis after grouping ICD-10-CA codes for main diagnoses codes to the Clinical Classifications Software. ${ }^{14}$

We defined hospital length of stay (LOS) as the time from admission to discharge and emergency department wait time as the time from triage by nursing staff to departure from the emergency department. There are no universally accepted definitions of "avoidable" admissions. ${ }^{1}$ Although it is challenging to determine observationally which admissions are avoidable, it is easier to estimate for patients who come from an LTC facility than for the general patient population. Hospital LOS for patients coming from an LTC facility is not prolonged by disposition planning, as patients already have a clear discharge destination: their LTC facility. Therefore, in patients from LTC facilities, the hospital LOS would be expected to correlate well with severity of illness and the need for hospital resources. We focused here on patients with admissions less than or equal to 72 hours rather than those with admissions longer than 72 hours on the basis of previous definitions in the literature for short stay units and avoidable admissions. ${ }^{15,16}$ 


\section{Modelling resource and physician staffing requirements for a virtual care strategy}

We estimated future requirements for outpatient diagnostic imaging and physician staffing for a virtual care strategy to reduce the frequency of patient transfers from LTC facilities to hospital. To be conservative in our estimates (i.e., to estimate the upper bound of future resources that would be required, on the basis of historical patterns of use), we modelled resource use by assuming that all "potentially avoidable" transfers from LTC could be diverted. We considered potentially avoidable transfers to include all patients who present to the emergency department and are returned to LTC without admission, which is approximately $50 \%$ of all LTC transfers according to published estimates in Ontario; ${ }^{18}$ and patients who are admitted for 72 hours or less. It is unlikely that any virtual physician consultation strategy supported by rapid access to the additional outpatient resources we describe (e.g., diagnostic imaging and intravenous medication) would be completely successful in eliminating all potentially avoidable transfers. Therefore, this model provides an upper bound on the outpatient resources required to support a virtual strategy, assuming needs are similar to historical patterns of use.

Given the relative lack of reliable data regarding COVID-19-related resource demands in Canada, we elected not to include estimates of this effect in our models. Additionally, we did not model important nonphysician resources that would be crucial to providing efficient and resident-centric care at LTC facilities. These include (but are not limited to) physiotherapists and occupational therapists, social workers, pharmacists and providers of skilled nursing care.

\section{Diagnostic imaging}

For each imaging modality, we determined the total number of scans across the 7 hospitals in each year from 2011 to 2016 for patients with hospital admissions of 72 hours or less. We multiplied this number by the time per scan, which was an estimated amount that comprised patient setup time, actual imaging time, and time to prepare the room for the next patient. This estimate varied by modality and body part imaged and was based on expert opinion (from a hospitalbased radiologist) because of the lack of published data around such times (Appendix 1, available at www.cmajopen. $\mathrm{ca} /$ content/8/3/E514/suppl/DC1). A linear regression was fit to the annual imaging volume and extrapolated to estimate an imaging volume for 2020. We also report a prediction interval, which provides a conservative estimate of uncertainty about the actual volume rather than a confidence interval. ${ }^{19}$ The weekly total for each modality was apportioned into hospital-specific estimates using the average proportion of imaging volume (in hours) from each hospital between 2011 and 2016.

We estimated the diagnostic imaging required for patients with hospital admissions of 72 hours or less on the basis of GEMINI, and we extrapolated these results to patients who had an emergency department visit but were not admitted.

\section{Length of stay}

Similar to our approach with diagnostic imaging, to estimate total LOS, we used yearly LOS (instead of yearly scan time) as the regression dependent variable. Hospital-specific proportions were also derived.

\section{Physician staffing}

We developed queuing models to estimate the number of physicians needed to staff a virtual assessment centre to serve the potential divertible population of each hospital..$^{20} \mathrm{We}$ focused on physicians in this model given an underlying assumption that physician assessment for an acute condition is one of the main objectives of sending residents of LTC facilities to a hospital emergency department. Queuing models are mathematical models used to study service systems such as call centres. They use inputs including the rate of arrivals to the system, the distribution of service completion times, and the number of servers to calculate performance metrics such as wait times in the system.

Starting with the triage times of patients who arrived at each hospital, we subtracted a random number of minutes drawn from a uniform distribution between 60 and $120 \mathrm{~min}$ utes to estimate the time of the original 911 call. For each year from 2011 to 2016, we then binned the call times into 1-hour increments and scaled the distribution so that the daily call volume that could be expected by a virtual centre was equal to twice the average historical daily call volume for admitted patients (based on a historical estimate that $50 \%$ of patients are not admitted). ${ }^{18}$ To estimate average daily call volume for 2020, we extrapolated via linear regression (using annual volumes from each year in the GEMINI data) the average daily call volume over all hospitals and used hospitalspecific proportions based on total call volume. We report a confidence interval because we are reporting on the average call volume. The distribution of estimated call arrival times was similar for each hospital (data not shown); Appendix 2 (available at www.cmajopen.ca/content/8/3/E514/suppl/DC1) shows the normalized distribution over all hospitals.

We assumed a Poisson distribution for arrivals and used thinning to simulate the nonstationary arrival process. ${ }^{21} \mathrm{On}$ the basis of expert opinion from internal medicine physicians who have provided virtual care assessments to LTC residents, we assumed that the duration of each assessment followed a triangle distribution with a centre at 45 minutes and minimum and maximum values at 15 and 75 minutes, respectively. We simulated 1000 days of operating the centre 24 hours a day and calculated the distribution of wait times (time from call until physician assessment begins) as a function of a fixed staffing level throughout the entire day. We conducted this queuing analysis for each hospital separately, on the basis of their individual volumes. To determine the relationship between call volume and waiting time for different staffing levels, we repeated the simulation for different levels of call volume.

\section{Statistical analysis}

Descriptive statistics were summarized using proportions, means with standard deviations (SDs) and medians with interquartile ranges (IQRs) where appropriate. For patients who were 
admitted from or discharged to an LTC facility, we compared patient characteristics, situational factors and utilization of hospital resources with standardized mean differences (SMDs), and we considered a value greater than 0.1 as a sign of meaningful difference between groups. ${ }^{17}$ Beyond testing for significance, SMDs also estimate the magnitude of the difference between groups.

GEMINI data go through a statistical and manual screening process that includes range checks and missing data checks during collection and harmonization. ${ }^{9}$ This process is iterative, and data are re-extracted when problems are identified. No additional data-cleaning steps were taken in the preparation of the data for this study.

\section{Ethics approval}

Each participating hospital obtained approval for the study from its research ethics board and authorized the use of data under a waiver of patient consent given the large size of the data set, the retrospective nature of the data and the limited risk to individual patients. In addition, the Unity Health Toronto Research Ethics Board permitted the central holding of each individual hospital's data set and use of this data for publication.

\section{Results}

The GEMINI cohort includes 245559 hospital admissions for which the patient was admitted to or discharged from the general internal medicine service between Apr. 1, 2010, and Oct. 31, 2017. For 235375 (96\%) of these admissions, the patient was admitted to the general internal medicine service from the emergency department; these admissions were the focus of this analysis. Residents of LTC facilities (aged $\geq 16 \mathrm{yr}$ ) accounted for $9.3 \%(n=21948)$ of all admissions to the general internal medicine service (Table 1). All 38 LTC facilities in Toronto had residents transferred to a GEMINI hospital during the study period.

\section{Patient characteristics}

Compared with other patients admitted to hospital, those from LTC facilities were older (aged 86.00 v. $70.00 \mathrm{yr}$ ), had higher medical acuity (LAPS value 26.48 v. 19.46) and higher Charlson Comorbidity Index scores (score $\geq 2$ in $62.5 \%$ of admissions of patients from LTC facilities $[n=13725]$ v. $41.9 \%$ of admissions of patients from other settings $[n=$ 89 518]) (Table 1). The percentage of admissions from LTC facilities that involved patients who had been admitted to the general medicine service of a GEMINI hospital in the prior 30 days was greater than the corresponding percentage of admissions from non-LTC settings (13.4\% [ $n=2913]$ v. $10.1 \%$ [ $n=21$ 101]) (Table 1 ).

\section{Resource use and outcomes for patients from long-term care facilities with short admissions}

Admissions for 72 hours or less accounted for about 24.1\% ( $n=$ 5297) of all admissions of patients from LTC facilities to the general internal medicine service of the study hospitals $(4.9 \%$ $[n=1084]$ for admissions of $<24 \mathrm{~h}$, and $19.2 \%[n=4213]$ for admissions of 24-72 h; Appendix 3, available at www.cmajopen. ca/content/8/3/E514/suppl/DC1). Approximately 25\% of

\begin{tabular}{|c|c|c|c|}
\hline \multirow[b]{2}{*}{ Characteristic } & \multicolumn{3}{|c|}{ No. (\%) of admissions* } \\
\hline & $\begin{array}{c}\text { Overall } \\
n=235375\end{array}$ & $\begin{array}{c}\text { Admissions of patients } \\
\text { from LTC facilities } \\
n=21948\end{array}$ & $\begin{array}{c}\text { Admissions of patients } \\
\text { from other settings } \\
n=213427\end{array}$ \\
\hline Age, yr, median (IQR) & $72.00(57.00-83.00)$ & $86.00(79.00-91.00)$ & $70.00(55.00-82.00)$ \\
\hline Sex, female & $117757(50.0)$ & $13209(60.2)$ & $104548(49.0)$ \\
\hline LAPS, mean \pm SD & $20.11 \pm 17.23$ & $26.48 \pm 18.08$ & $19.46 \pm 17.00$ \\
\hline Charlson Comorbidity Index score $\geq 2$ & $103243(43.9)$ & $13725(62.5)$ & $89518(41.9)$ \\
\hline Time in ED, h, median (IQR) & $14.67(9.90-23.65)$ & $16.00(10.18-26.38)$ & $14.47(9.83-23.23)$ \\
\hline Admission to GIM from ED & $209159(88.9)$ & $18915(86.2)$ & $190244(89.1)$ \\
\hline \multicolumn{4}{|l|}{ Time of admission } \\
\hline Weekday & $175121(74.4)$ & $16034(73.1)$ & $159087(74.5)$ \\
\hline Daytime & $54136(23.0)$ & $5359(24.4)$ & $48777(22.9)$ \\
\hline \multicolumn{4}{|l|}{ Time of triage } \\
\hline Weekday triage & $177298(75.3)$ & $16195(73.8)$ & $161103(75.5)$ \\
\hline Daytime & $129744(55.1)$ & $12373(56.4)$ & $117371(55.0)$ \\
\hline Admitted to GIM in previous $30 \mathrm{~d}$ & $24014(10.4)$ & $2913(13.4)$ & $21101(10.1)$ \\
\hline
\end{tabular}


admissions for 72 hours or less were for patients who had a cardiorespiratory reason for their admission (Appendix 4, available at www.cmajopen.ca/content/8/3/E514/suppl/DC1).

Nearly all patients with LOS of 72 hours or less received laboratory testing (99.8\% of admissions, $n=5284$ admissions) (Table 2). Among diagnostic imaging modalities, plain radiography (86.9\% of admissions, $n=4604$ admissions) and CT (41.5\% of admissions, $n=2197$ admissions) were the most commonly used (Table 2). Ultrasonography was used in $12.1 \%$ of admissions ( $n=642$ admissions), and MRI and interventional radiology were used in less than $2 \%$ of admissions (Table 2). Patients in $81.2 \%$ of admissions $(n=4300$ admissions) needed IV medications (Table 2).

The mortality rate was high among patients with an admission of less than 24 hours $(26.3 \%$ of admissions for less than 24 hours resulted in death of the patient [Appendix 3]). The 5 most common discharge diagnoses of patients admitted from LTC facilities were urinary tract infection, pneumonia, aspiration pneumonitis, heart failure, and delirium or dementia.

\section{Modelling staffing and resource requirements for a virtual care strategy}

\section{Diagnostic imaging and length of stay}

For an average hospital, the weekly demand for outpatient imaging would be 2.6 ultrasounds, 11.9 CT scans and 23.9 radiographs (Appendix 5, available at www.cmajopen.ca/content/8/3/E514/suppl/DC1). Patients who were admitted would require 5.7 bed-days per week per hospital.

\section{Virtual assessment centre staffing}

The average daily volume of urgent medical virtual assessments would range from 2.0 to 5.8 per hospital. With a single physician staffing a virtual call centre, the average wait time would exceed 15 minutes once the daily call volume exceeded 10 calls (Figure 1). With 2 physicians, the average wait time would remain below 16 minutes for up to 30 calls per day. The 90th percentile of the wait time with 2 physicians would

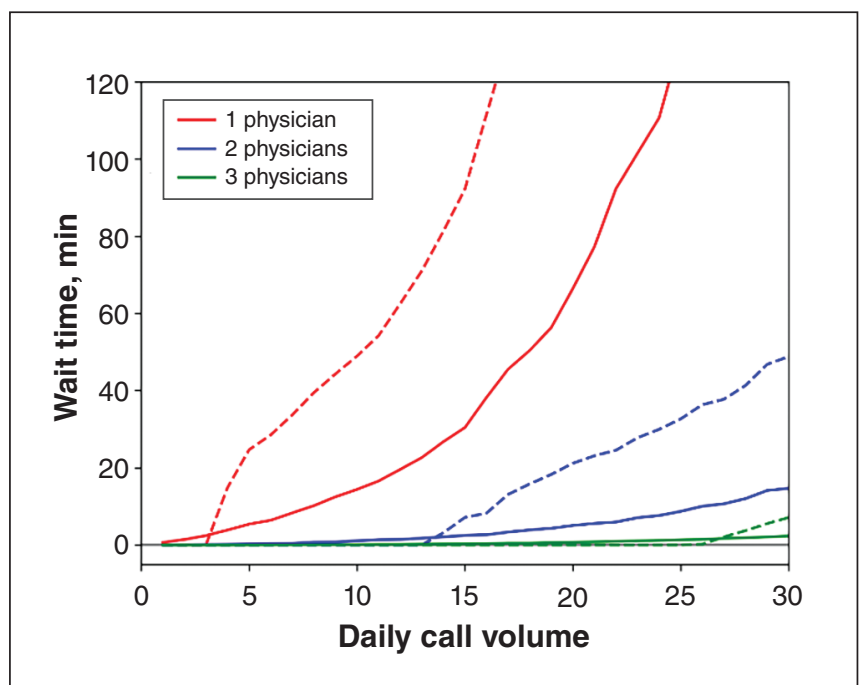

Figure 1: Average waiting times (dashed lines) and 90th percentile waiting times (solid lines) by daily call volume for virtual assessment centres staffed by 1,2 or 3 physicians 24 hours a day.

\begin{tabular}{|c|c|c|c|c|}
\hline \multirow[b]{2}{*}{ Characteristic } & \multicolumn{3}{|c|}{ No. $(\%)$ of admissions } & \multirow[b]{2}{*}{ SMD } \\
\hline & $\begin{array}{c}\text { All admissions } \\
n=21948\end{array}$ & $\begin{array}{c}\text { Admissions for patients } \\
\text { with LOS } \leq 72 \mathrm{~h} \\
n=5297\end{array}$ & $\begin{array}{l}\text { Admissions for patients } \\
\text { with LOS }>72 \mathrm{~h} \\
n=16651\end{array}$ & \\
\hline Length of stay, d, median (IQR) & $5.75(3.12-10.80)$ & $1.88(1.23-2.57)$ & $7.62(4.90-13.34)$ & 0.625 \\
\hline IV medication & $19332(88.1)$ & $4300(81.2)$ & $15032(90.3)$ & 0.262 \\
\hline Laboratory testing & 21933 (99.9) & $5284(99.8)$ & $16649(100.0)$ & 0.065 \\
\hline \multicolumn{5}{|l|}{ Diagnostic imaging } \\
\hline Any plain radiography & $20332(92.6)$ & $4604(86.9)$ & $15728(94.5)$ & 0.262 \\
\hline Any CT & $11571(52.7)$ & $2197(41.5)$ & $9374(56.3)$ & 0.300 \\
\hline Any ultrasonography & $5794(26.4)$ & $642(12.1)$ & $5152(30.9)$ & 0.470 \\
\hline Any MRI & $920(4.2)$ & $46(0.9)$ & $874(5.2)$ & 0.256 \\
\hline Any interventional radiology & $1716(7.8)$ & $90(1.7)$ & $1626(9.8)$ & 0.352 \\
\hline $\mathrm{RBC}$ transfusion & $1707(7.8)$ & $233(4.4)$ & $1474(8.9)$ & 0.180 \\
\hline Endoscopy & $1234(5.6)$ & $164(3.1)$ & $1070(6.4)$ & 0.157 \\
\hline ICU admission & $1605(7.3)$ & $131(2.5)$ & $1474(8.9)$ & 0.279 \\
\hline Death & $2710(12.3)$ & $772(14.6)$ & $1938(11.6)$ & 0.087 \\
\hline Readmission to GIM in $30 \mathrm{~d}$ & $2698(14.1)$ & $578(12.8)$ & $2120(14.5)$ & 0.048 \\
\hline
\end{tabular}


exceed 15 minutes once the call volume exceeded 17 calls. With 3 physicians, the 90th percentile wait time would remain below 9 minutes up to a call volume of 30 .

For the 7 study hospitals and their specific call volumes, 1 physician working for each hospital (i.e., 7 physicians working simultaneously across the 7 hospitals) would be able to maintain a 90th percentile wait time below 27.1 minutes (Appendix 6, available at www.cmajopen.ca/content/8/3/E514/ suppl/DC1). However, if the total call volume across these 7 hospitals was pooled, roughly 26.9 calls per day, then 2 physicians at a single virtual assessment centre would achieve a 90th percentile wait time of 38.1 minutes (Figure 1, Appendix 7, available at www.cmajopen.ca/content/8/3/E514/suppl/DC1).

The busiest 12 hours of the day (i.e., 7 am to $7 \mathrm{pm}$ ) accounted for $73.4 \%$ of the daily call volume (Appendix 2).

\section{Interpretation}

This paper offers 2 major insights about the diagnostic and therapeutic resources and physician staffing volumes that may be required to support the delivery of urgent medical care to the residents of LTC facilities to reduce transfers to hospital. First, the outpatient diagnostic imaging resources that are required are probably on a scale that is achievable using existing outpatient services (3 ultrasounds, 12 CT scans and 24 radiographs per hospital per week). Second, if a virtual physician consultation service were established, consolidation to centralized hubs would greatly reduce the number of physicians required to staff these services at any given time while still enabling the service to maintain safe response times. A centralized hub with 2-3 physicians would provide service equal in efficiency to decentralized hubs with 7 physicians (1 per hospital) in our study.

If residents of LTC facilities can avoid being transferred to hospital, they will not face the risk of developing COVID-19 associated with possible exposure during assessment and transportation by emergency medical services, in the emergency department or on hospital wards. Such nosocomial exposure has been a noted mechanism of spread for other respiratory viral pathogens such as seasonal influenza viruses and the coronavirus that causes severe acute respiratory syndrome (SARS). ${ }^{4,5}$ Our data demonstrate that LTC residents have higher 30-day readmission rates than typical general medicine patients. This is particularly relevant given that approximately $80 \%$ of COVID-19 spread occurs through asymptomatic carriers. ${ }^{22}$ Through nosocomial exposure, an LTC resident could become an asymptomatic COVID-19 vector and precipitate an outbreak among the other residents of their facility. More generally, reducing avoidable transfers from LTC facilities to hospital limits the risk of hospitalrelated iatrogenic harm or adverse events when patients are transferred back to their LTC facility. ${ }^{23,24}$

Reducing hospital transfers of LTC residents may also be an important strategy to protect emergency department and ward capacity if there is a surge in admissions related to COVID-19. Our data demonstrate that residents of LTC have higher readmission rates within 30 days than patients typically admit- ted to the general internal medicine service. Furthermore, COVID-19 outbreaks in LTC facilities could also become barriers to transfer back to a patient's home facility.

Our data suggest that nearly all admissions to hospital of patients from LTC facilities involve laboratory testing and more than $88 \%$ involve the use of IV medication. We therefore did not model the requirement for these services, as any urgent medical care would probably require onsite phlebotomy and the ability to deliver IV medication. Moreover, to deliver high-quality urgent medical care in LTC facilities, the most important resource is local primary care and nursing. In addition, given that approximately $25 \%$ of patients who had a hospital LOS of 72 hours were admitted for a cardiorespiratory reason, onsite electrocardiography and the ability to provide supplemental oxygen would be essential components of any service that provided diagnosis and management.

Diagnostic imaging is crucial to the assessment process for many patients requiring acute care but may not be readily portable and therefore is unlikely to be accessible onsite at most LTC facilities. Even in an ambitious scenario wherein the provision of virtual care successfully prevented all transfers of patients who would not have been admitted or would have had short admissions (about $60 \%$ of total transfers between these 2 groups), it is probably possible to meet the demand for outpatient radiographs (estimated at 23.9 per hospital per week) and CT scans (estimated at 11.9 per hospital per week) if resources were marshalled through outpatient imaging facilities associated with hospitals or other local imaging clinics.

Our model suggests that a single physician providing virtual consultations for a given hospital would be able to respond to typical calls from LTC facilities within an acceptable time frame ( $<15 \mathrm{~min}$ ) for up to 10 calls per day, a number that is higher than typical transfer volumes. However, economies of scale can result from aggregating virtual calls. For example, in the 7 hospitals in GEMINI, a consolidated virtual service staffed by just 2 or 3 physicians at any given time could reach the same 90 th percentile wait time as 7 individual physicians working simultaneously at each hospital.

Our model focuses on urgent medical consultation and does not account for the need for various physician and allied health specialties that would be part of a broad and fully patient-centred virtual consultation service. For example, $26 \%$ of patients admitted for less than 24 hours die within hospital, and this highlights the probable need for palliative services in addition to general consultative services. Moreover, decisions about how to deploy virtual assessment services should consider factors other than efficiency, such as developing and leveraging institutional relationships and local partnerships to facilitate quality improvement, which may be easier with some degree of decentralization. Finally, many of the most common reasons for transfer are chronic conditions (e.g., heart failure) for which high-quality preventive care may reduce the frequency of acute exacerbations. ${ }^{25}$ These represent important conditions where transfers may be avoidable, before acute care is required.

The results of this analysis helped inform the design of a rapidly evolving care strategy in response to COVID-19 that 
is being deployed across a large number of LTC facilities in the Toronto region called LTC+ (https://ltcplus.ca). ${ }^{26} \mathrm{LTC}+$ aims to provide acute care for residents of LTC facilities who have medical issues at their facility and reduce the need for hospital transfer.

\section{Limitations}

This study has a number of limitations. There is no wellaccepted definition of an avoidable transfer from an LTC facility to hospital. Prior attempts to provide proxies for avoidability have used measures such as ambulatory care sensitive conditions, low-acuity admissions or short admissions. ${ }^{15,18} \mathrm{We}$ provide an upper bound on the total resources that may be required, but it is unlikely that all of these patients truly represent avoidable transfers. Our models are based on admissions to general medicine whereas many transfers from LTC facilities to hospital may be for issues like injuries requiring other types of physician or nonphysician services. However, our focus was on delivering urgent medical services, which is particularly salient in the setting of COVID-19. Our modelling is based on estimates of historical use. It is possible that in the setting of COVID-19 outbreaks, there will be increased demand for virtual urgent medical services to LTC facilities, especially palliative care.

Our modelling does not consider the technical demands (e.g., infrastructure and credentialling) that would be required to support a virtual care service, and these represent important process barriers to a rapid implementation of a virtual consultation service. Such factors may also influence decisions regarding centralization versus decentralization. Our models use resource demands from patients with short admissions to extrapolate to those who are transferred from LTC facilities to emergency departments but not admitted. This probably represents an overestimate of the resources required, especially given the differences in the primary referral reason for patients who are admitted versus not admitted. ${ }^{18}$

This paper does not model the increase in staffing resources at the LTC facility that would be required to support virtual care assessments and facilitate implementation of treatment recommendations. Our sample is derived from patients transferred to hospital from LTC facilities in the Toronto region and may not be generalizable to other regions. However, nearly $60 \%$ of all COVID-19 cases in Ontario are from the Toronto region, making these estimates useful in understanding a major component of the provincial burden of disease. ${ }^{27}$

Given wide variation in the estimates of COVID-19 cases and the rapid changes in these estimates, we elected not to include projections based on COVID rates but rather kept our focus on the types of resources required to support care at LTC facilities. Over an extended period, a patient may have been admitted and discharged many times. However, we do not believe this clustering affects our interpretation of our findings as we modelled the resource demands for each episode of care. For example, if a given patient were transferred to hospital from an LTC facility on 2 occasions months apart, each episode of care would require resources (blood work, physician consultation, etc.) and that is what we modelled.

\section{Conclusion}

This study models the resources required if a virtual strategy for urgent medical care were adopted to reduce transfer of patients from LTC facilities to hospital. These results have directly informed the design of a rapidly evolving Ontario Health program called LTC+ that is providing a virtual consultation service to all LTC facilities in the Toronto region, and these findings may have application across various jurisdictions in light of a potential surge in COVID-19 cases.

\section{References}

1. Gruneir A. "Avoidable" emergency department transfers from long-term care homes: a brief review. Healthc Q 2013;16:13-5.

2. Guan W-J, Ni Z-Y, Hu Y, et al.; China Medical Treatment Expert Group for Covid-19. Clinical characteristics of coronavirus disease 2019 in China. $N$ Engl 7 Med 2020;382:1708-20.

3. McMichael TM, Clark S, Pogosjans S, et al.; Public Health - Seattle \& King County, EvergreenHealth, and CDC COVID-19 Investigation Team. COVID-19 in a long-term care facility - King County, Washington, February 27-March 9, 2020. MMWR Morb Mortal Wkly Rep 2020;69:339-42.

4. Murti M, Whelan M, Friedman L, et al. Influenza outbreaks in Ontario hospitals, 2012-2016. Can Commun Dis Rep 2018;44:201-5.

5. Varia M, Wilson S, Sarwal S, et al.; Hospital Outbreak Investigation Team. Investigation of a nosocomial outbreak of severe acute respiratory syndrome (SARS) in Toronto, Canada. CMA7 2003;169:285-92.

6. Verma AA, Guo Y, Kwan JL, et al. Patient characteristics, resource use and outcomes associated with general internal medicine hospital care: the General Medicine Inpatient Initiative (GEMINI) retrospective cohort study. CMA7 Open 2017;5:E842-9.

7. DAD Data Elements 2018-2019 [tables]. Ottawa: Canadian Institute for Health Information. Available: www.cihi.ca/sites/default/files/document/ dad-data-elements-2018-en-web.pdf (accessed 2020 Mar. 6).

8. NACRS Data Elements 2018-2019 [tables]. Ottawa: Canadian Institute for Health Information. Available: www.cihi.ca/sites/default/files/document/ nacrs-dataelements-2018-2019-en.pdf (accessed 2020 Mar. 6).

9. Pasricha SV, Jung HY, Kushnir V, et al. Assessing the quality of clinical and administrative data extracted from hospitals: the General Medicine Inpatient Initiative (GEMINI) experience. medRxiv 2020 Mar. 18. doi: $10.1101 / 2020.03 .16 .20036962$

10. Ontario Ministry of Health. Ministry reports. Master numbering system. Toronto: Ontario Ministry of Health; 2020. Available: www.health.gov.on.ca/ en/common/ministry/publications/reports/master_numsys/master_numsys.aspx (accessed 2020 Mar. 6).

11. Quan H, Li B, Couris CM, et al. Updating and validating the Charlson Comorbidity Index and score for risk adjustment in hospital discharge abstracts using data from 6 countries. Am 7 Epidemiol 2011;173:676-82.

12. Escobar GJ, Greene JD, Scheirer P, et al. Risk-adjusting hospital inpatient mortality using automated inpatient, outpatient, and laboratory databases. Med Care 2008;46:232-9.

13. Canadian Institute for Health Information. Canadian coding standards for version 2018 ICD-10-CA and CCI. Ottawa: Canadian Institute for Health Information; 2018. Available: https://secure.cihi.ca/estore/productSeries.htm?pc=PCC189 (accessed 2020 Mar. 6).

14. Agency for Healthcare Research and Quality. Healthcare Cost and Utilization Project (HCUP). Clinical Classifications Software (CCS) for ICD-10-PCS (beta version). Rockville (MD): Agency for Healthcare Research and Quality; updated 2019 Nov. 5. Available: www.hcup-us.ahrq.gov/toolssoftware/ccs10/ ccs10.jsp (accessed 2020 Mar. 6).

15. Verma AA, Guo Y, Kwan JL, et al. Characteristics of short general internal medicine hospital stays: a multicentre cross-sectional study. CMAf Open 2019;7:E47-54.

16. Galipeau J, Pussegoda K, Stevens A, et al. Effectiveness and safety of shortstay units in the emergency department: a systematic review. Acad Emerg Med 2015;22:893-907.

17. Austin PC. Using the standardized difference to compare the prevalence of a binary variable between two groups in observational research. Commun Stat Simul Comput 2009;38:1228-34. doi: 10.1080/03610910902859574.

18. Gruneir A, Bell CM, Bronskill SE, et al. Frequency and pattern of emergency department visits by long-term care residents - a population-based study. $\mathcal{F}$ Am Geriatr Soc 2010;58:510-7.

19. Patel JK. Prediction intervals - a review. Commun Stat Theory Methods 1989;18:2393-465. doi: 10.1080/03610928908830043.

20. Nelson B. Foundations and methods of stochastic simulation: a first course. New York: Springer; 2013.

21. Gerhardt I, Nelson BL. Transforming renewal processes for simulation of nonstationary arrival processes. INFORMS 7 Comput 2009;21:630-40. doi: $10.1287 /$ ijoc. 1080.0316 
22. Li R, Pei S, Chen B, et al. Substantial undocumented infection facilitates the rapid dissemination of novel coronavirus (SARS-CoV2). Science 2020; 368:489-93.

23. Ouslander J. Strategies to reduce potentially avoidable hospitalisations among long-term care facility residents. BM7 Qual Saf 2019;28:515-9.

24. Kapoor A, Field T, Handler S, et al. Adverse events in long-term care residents transitioning from hospital back to nursing home. 7AMA Intern Med 2019; 179:1254-61.

25. Heckman GA, Shamji AK, Ladha R, et al. Heart failure management in nursing homes: a scoping literature review. Can $\mathcal{F}$ Cardiol 2018;34:871-80.

26. LTC+ [main page]. Toronto: Women's College Hospital. Available: https:// ltcplus.ca/ (accessed 2020 Mar. 6).

27. Ontario Agency for Health Protection and Promotion (Public Health Ontario). Epidemiologic summary: COVID-19 in Ontario - Fanuary 15, 2020 to April 24, 2020. Toronto: Queen's Printer for Ontario; 2020.

Affiliations: Division of General Internal Medicine (Razak, Verma), St. Michael's Hospital; Department of Medicine (Razak, Moser, LapointeShaw, Tang, Kwan, Weinerman, Rawal, Shojania, Bhatia, Mukerji, Kapral, Morgan, Verma), University of Toronto; Li Ka Shing Knowledge Institute (Razak, Shin, Pogacar, Jung, Kushnir, Mak, Fralick, Chan, Verma), St. Michael's Hospital; Department of Mechanical and Industrial Engineering (Pogacar, Chan), University of Toronto; Women's College Hospital Institute for Health Systems Solutions and Virtual Care (Pus, Martin, Bhatia, Agarwal, Mukerji), Women's College Hospital; Baycrest Geriatric Health Care System (Moser); Division of General Internal Medicine (Lapointe-Shaw, Rawal, Fralick), University Health Network, Toronto, Ont.; Trillium Health Partners (Tang), Mississauga, Ont.; Department of Medicine (Kwan, Fralick, Morgan), Mount Sinai
Hospital; Sunnybrook Health Sciences Centre (Weinerman, Shojania, Wong); Department of Family and Community Medicine (Martin, Agarwal), University of Toronto, Toronto, Ont.

Contributors: Fahad Razak, Lauren Lapointe-Shaw, Terence Tang, Janice Kwan, Shail Rawal, Vladyslav Kushnir, Denise Mak, Michael Fralick and Amol Verma acquired the data. Fahad Razak, Saeha Shin, Frances Pogacar, Hae Young Jung, Timothy Chan and Amol Verma analyzed and interpreted the data and drafted the manuscript. All of the authors contributed to the study conception and design, revised the manuscript critically for important intellectual content, approved the final version to be published and agreed to be accountable for all aspects of the work. Saeha Sim and Frances Pogacar contributed equally to this work. Brian Wong, Timothy Chan and Amol Verma are joint senior authors.

Funding: Fahad Razak is supported by an award from the Mak Pak Chiu and Mak-Soo Lai Hing Chair in General Internal Medicine, University of Toronto.

Data sharing: The data from this study are held securely by GEMINI at Unity Health Toronto. Under the data-sharing agreement involving the affiliated hospitals, the data are not accessible to others. Analysis using these data must be performed by a GEMINI analyst or approved user within the secure data environment provided by Unity Health.

Supplemental information: For reviewer comments and the original submission of this manuscript, please see www.cmajopen.ca/content/8/3/ E514/suppl/DC1. 